\title{
Overweight as an Additional Risk Factor for Colorectal Neoplasia in Lean Population
}

\author{
Sureeporn Jangsirikul ${ }^{1}$, Wasinee Promratpan ${ }^{1}$, Satimai Aniwan ${ }^{1}$, Natanong \\ Kongtub $^{1}$, Naruemon Wisedopas ${ }^{2}$, Pinit Kullavanijaya ${ }^{1}$, Rungsun Rerknimitr ${ }^{1 *}$
}

\begin{abstract}
Background: Overweight in Thailand is not as common as in Western countries. We sought to evaluate overweight as the additional risk factor that can increase the prediction of colorectal neoplasia (CRN) detection in Thais apart from the Asia-Pacific Colorectal Screening (APCS) score. Methods: We prospectively enrolled asymptomatic 338 subjects who underwent screening colonoscopy between November 2016 and September 2017. All risk factors according to APCS, BMI and the presence of metabolic syndrome were collected. Overweight was defined as BMI $\geq 23 \mathrm{~kg} / \mathrm{m}^{2}$. By APCS score, subjects were categorized into 1) high-risk and 2) average-risk. Using the combination of APCS score and overweight, subjects were stratified into 4 groups; high-risk with overweight (G1), average-risk with overweight (G2), high-risk with normal weight (G3) average-risk and with normal weight (G4). Logistic regression analysis was used to estimate the risk of detecting CRN. Results: The prevalence of CRN in the high-risk subjects was higher than that of in the average-risk subjects (49\%vs.32\%; OR, 2.00; $95 \% \mathrm{CI}, 1.17-3.41)$. After adjustment for APCS risk factors and metabolic syndrome, overweight significantly increased the risk of detecting CRN (OR, 2.52; 95\%CI, 1.57-4.05). Among the 4 groups, the detection rates of $\mathrm{CRN}$ were significantly different (G1 $=64 \%, \mathrm{G} 2=40 \%, \mathrm{G} 3=32 \%$ and $\mathrm{G} 4=21 \%, \mathrm{p}<0.01$ ). The relative risk of detecting CRN increased when $\mathrm{G} 1$ (OR 6.49; 95\%CI, 2.87-14.67), and G2 $(2.42 ; 1.39-4.21)$ were compared with G4. Conclusions: In addition to the APCS score, overweight is an independent risk factor for detecting CRN. In Thai population, combining overweight and APCS score may be useful to improve the prediction for CRN.
\end{abstract}

Keywords: Colorectal adenoma- colorectal cancer screening- colonoscopy- risk stratification- body mass index

Asian Pac J Cancer Prev, 20 (1), 105-111

\section{Introduction}

Incidence of colorectal cancer (CRC) has been increasing in Asia and also in Thailand (Sung et al., 2005). Although colonoscopy is the best single colonic examination and provides an opportunity for polypectomy, its cost and nature of the technique that requiring endoscopist attribute to tremendous burden on healthcare systems in the resource-limited countries.

To reduce the cost and workload of colonoscopy, the updated Asia-Pacific Consensus on CRC proposed a risk stratified approach to select a high-risk subject for an early colonoscopy (Sung et al., 2015). The Asia-Pacific Colorectal Screening (APSC) score has been developed across 11 countries in Asia and Pacific region (Yeoh et al., 2011). It has been validated in Asian and Western population (Aniwan et al., 2015; Corte et al., 2016; Aniwan et al., 2017; Quach et al., 2018). The score that composed of 4 traditional CRC risk factors; age, sex, family history of CRC and smoking, ranges from score 0 to 7. These scores are grouped into low-risk, average-risk, and high-risk (Table 1). When compared with the low-risk group, the relative risk of detecting advanced CRN was 2.6-fold in the average-risk and 4.3-fold in the high-risk (Yeoh et al., 2011)

In addition to the traditional CRC risk factors, overweight was found as an environmental factor for developing CRC (Harriss et al., 2009). The proposed mechanism is an increase in insulin-like growth factor, leptin, vascular endothelial growth factor leading patients to chronic inflammation and cancer (Stattin et al., 2004; Byrne et al., 2005; Pollak, 2008; Braun et al., 2011; Hursting and Hursting, 2012). A recent meta-analysis including Western and Asian studies showed the positive association between overweight and the detection rate of colorectal adenoma (Okabayashi et al., 2012). Over the last decade, overweight has become a global burden throughout world (Kelly et al., 2008). Although

${ }^{I}$ Division of Gastroenterology, Department of Medicine, Faculty of Medicine, Chulalongkorn University and King Chulalongkorn Memorial Hospital, Thai Red Cross, ${ }^{2}$ Department of Pathology, Faculty of Medicine, Chulalongkorn University, Bangkok, Thailand. *For Correspondence: ercp@live.com 
overweight in Thailand is not as common as in Western countries, one-fifth of Asian population were overweight in 2005 and the estimated prevalence of overweight has been projected to approximately $50 \%$ of Asian population by 2030 (Kelly et al., 2008). Since the original APCS score did not include overweight as a parameter, hence the potential increase in the prediction of $\mathrm{CRN}$ detection by adding overweight as additional factor to the APCS score in Thai population is uncertain. To confirm this hypothesis, this study was taken to evaluate the association between the combination of overweight and the APCS score and the detection rate of CRN in asymptomatic Thais who presented for CRC screening.

\section{Materials and Methods}

\section{Study population}

We conducted a prospective cross-sectional study including consecutive asymptomatic subjects who attended the CRC screening clinic at the King Chulalongkorn Memorial Hospital between 1 November 2016 and 30 September 2017. Asymptomatic subjects, aged 50 to 75 years were recruited. Exclusion criteria were history of $\mathrm{CRC}$, history of inflammatory bowel disease and family history of hereditary CRC ( $\geq 2$ first-degree relatives with $\mathrm{CRC}$ or $\geq 1$ first degree relative diagnosed with $\mathrm{CRC}$ before age of 60 years). All subjects provided written informed consent. This study was approved by the Chulalongkorn Institutional Review Board.

\section{Data collection}

All subjects were interviewed to determine their clinical risk score by the research assistant (N.K.). We stratified subjects into 2 groups according to APCS score; 1) average-risk defined as APCS score of 2-3 and 2) high-risk defined as APCS score of 4-7 (Table 1) (Yeoh et al., 2011). Data on demographic characteristics, body weight, height, waist circumference, medical history were collected. Serum triglyceride, serum high-density lipoproteins (HDL) cholesterol, and serum fasting glucose at colonoscopy screening or within one year before were collected. Using body mass index (BMI) cutoff at $23 \mathrm{~kg} / \mathrm{m}^{2}$ for Asian population (WHO, 2004), we categorized subjects into 1 ) overweight (BMI $\geq 23$ $\left.\mathrm{kg} / \mathrm{m}^{2}\right) ; 2$ ) normal weight (BMI $<23 \mathrm{~kg} / \mathrm{m}^{2}$ ). According to metabolic syndrome diagnostic criteria of National Cholesterol Education Program, Adult Treatment Panel III , metabolic syndrome was defined as meeting at least three of the following criteria; 1) waist circumference $\geq 90$ $\mathrm{cm}$ for Asian men or $\geq 80 \mathrm{~cm}$ for Asian women,; 2) serum triglyceride $\geq 150 \mathrm{mg} / \mathrm{dL}$; 3 ) HDL cholesterol $<40 \mathrm{mg} / \mathrm{dL}$ for men or $<50 \mathrm{mg} / \mathrm{dL}$ for women; 4) fasting glucose $\geq 110$ $\mathrm{mg} / \mathrm{dL}$ (National Cholesterol Education Program Expert Panel on Detection, 2002).

Bowel preparation and total colonoscopy were performed as described previously (Aniwan et al., 2016). All detected polyps were removed. Each polyp was measured by open biopsy forceps, 7-mm in diameter. Polyp was classified by pathology to 1) colorectal neoplasia 2) colorectal non-neoplasia. Colorectal neoplasia (CRN) was defined as adenoma (i.e. tubular adenoma, villous adenoma, tubulovillous adenoma, sessile/traditional serrated adenoma) or CRC. CRN was further classified as advanced $\mathrm{CRN}$ and non-advanced CRN. Advanced CRN was defined as adenoma with size $\geq 10 \mathrm{~mm}$. or high grade dysplasia or villous (at least $25 \%$ ) or CRC.

\section{Statistical analysis}

To compare categorical variables, Chi-square test or Fisher exact test was used. To compare continuous variables, Student's t-test or Mann-Whitney U-test was used. Logistic regression analysis was used to assess the association between the potential risk factors (i.e. age, sex, family history of CRC, smoker, overweight and metabolic syndrome) and CRN. Any variables with $\mathrm{p}<0.10$ in the univariate analysis were included in the multivariate analysis to estimate the odds ratio (OR) and $95 \%$ confidence interval $(\mathrm{CI})$. In order to evaluate whether the combination between overweight and the APCS score improve the detection of CRN, subjects were stratified by 4 groups; 1) subject with high-risk and overweight group (G1); 2) subject with average-risk and overweight group (G2); 3) subject with high-risk and normal weight group (G3); 4) subject with average-risk and normal weight group (G4).

We hypothesized that subject with high-risk and overweight would have the highest prevalence of CRN. In Thailand, the prevalence of CRN was 27\% (Aniwan et al., 2015) and the prevalence of the high-risk was $29 \%$ (Aniwan et al., 2017). Assuming the detection rate of CRN in subject with average-risk and normal body weight was $20 \%$ and that of subject with high-risk and overweight was $50 \%$. We estimated the prevalence of subjects with highrisk and overweight was $12 \%$. Therefore, a sample size of at least 300 subjects was required to detect $30 \%$ difference with a power of $80 \%$ at a two-sided significance level of 0.05 . Statistical analyses were performed by using SPSS statistical software (version 23.0; PSS Inc, Chicago III).

\section{Results}

A total of 338 subjects were enrolled. All subjects had a complete colonoscopy. Mean age was $62.7 \pm 8.6$ years. Two hundred and nine subjects (62\%) were female. Mean BMI was $23.9 \pm 3.7 \mathrm{~kg} / \mathrm{m}^{2}$. Of 338 subjects, overweight was found in 192 subjects ( $57 \%$ ) and metabolic syndrome was diagnosed in 136 subjects $(40 \%)$. The overall detection rates of CRN, advanced CRN, and CRC were 120 (36\%), $25(7 \%)$, and $2(0.6 \%)$, respectively. Demographic characteristics are shown in Table 2.

\section{Overweight and metabolic syndrome}

The detection rates of CRN and advanced CRN in subjects with overweight were higher than those of subjects with normal weight (for CRN 44\% vs. 24\%; $\mathrm{p}<0.01$ and for advanced CRN $12 \%$ vs. $2 \% ; \mathrm{p}<0.01$, respectively) The detection rates of CRN and advanced CRN between subjects with metabolic syndrome and those without metabolic syndrome were not significantly different (for CRN $38 \%$ vs. $34 \%$; $\mathrm{p}=0.39$ and for advanced CRN 7\% vs. 7\%; $\mathrm{p}=1.00$, respectively). Table 3 shows the univariate and multivariate analysis for the 
Table 1. Asia-Pacific Colorectal Screening Scoring System for Predicting the Risk for Colorectal Neoplasm

\begin{tabular}{lc}
\hline Variables Criteria & Points \\
\hline Age & \\
$\quad<50$ & 0 \\
$50-69$ & 2 \\
$\quad \geq 70$ & 3 \\
Sex & \\
$\quad$ Female & 0 \\
Male & 1
\end{tabular}

Family history of colorectal cancer in a first-degree relative

$$
\text { Absent }
$$

Present

Smoking

Never

Current or past

low-risk group, score of 0-1; average-risk group, score of 2-3; highrisk group, score of 4-7

association between potential risk factors and the risk of CRN detection. In multivariate logistic regression, after adjusting for traditional risk factors for CRC, there was statistically significant association between overweight and the CRN detection (OR, 2.57; 95\% CI, 1.57-4.20). When the model of analysis was adjusted with the highrisk score, overweight remained an increased risk for the CRN detection (OR, 2.68; 95\% CI, 1.65-4.36). For advanced $\mathrm{CRN}$, the detection rates of advanced CRN were numerically higher in subjects with traditional CRC risk factors as compared to subjects without traditional CRC risk factors ( $p>0.05$ for all comparisons). Only overweight significantly increased the risk for detecting advanced CRN (OR, 6.17; 95\%CI, 1.81-21.0) (Supplement Table 1).

\section{APCS scoring system}

Stratification by APCS score, there were 268 subjects $(79 \%)$ with average-risk and 70 subjects $(21 \%)$ with
Table 2. Demographic Characteristics of 338 Subjects

\begin{tabular}{lc}
\hline Variables & $\mathrm{N}=338$ \\
\hline Age (year), mean (SD) & $62.7(8.6)$ \\
$50-69$ years (n, \%) & $272(80 \%)$ \\
$\geq 70$ years (n, \%) & $66(20 \%)$ \\
Male (n, \%) & $129(38 \%)$ \\
First degree relative with colorectal cancer (n, \%) & $28(8 \%)$ \\
Current or former smoker (n, \%) & $39(12 \%)$ \\
Body mass index (kg/m $\left.{ }^{2}\right)$, mean (SD) & $23.9(3.7)$ \\
BMI $\geq 23 \mathrm{~kg} / \mathrm{m}^{2}(\mathrm{n}, \%)$ & $192(57 \%)$ \\
Metabolic syndrome ${ }^{\mathrm{a}}(\mathrm{n}, \%)$ & $136(40 \%)$ \\
APCS score ${ }^{\mathrm{b}}(\mathrm{n}, \%)$ & \\
Average risk & $268(79 \%)$ \\
High risk & $70(21 \%)$ \\
Prevalence of colorectal neoplasia (n, \%) & \\
All colorectal neoplasia & $120(36 \%)$ \\
Advanced colorectal neoplasia & $25(7 \%)$ \\
Cancer & $2(0.6 \%)$ \\
\hline
\end{tabular}

SD, standard deviation; APCS score, Asia-Pacific Colorectal Screening score; a, Metabolic syndrome was defined according to National Cholesterol Education Program (NCEP), Adult Treatment Panel III (ATP III); b, Average risk was defined as an APCS score of 2-3, and high risk was defined as an APCS score of 4-7.

high-risk, respectively. The detection rate of CRN was $49 \%$ for subjects with high-risk and was $32 \%$ for subjects with average-risk $(\mathrm{p}=0.01)$. The detection rate of advanced CRN was $9 \%$ for subjects with high-risk and $7 \%$ for subjects with average-risk $(p=0.67)$. Subjects with high-risk significantly had an increased risk for CRN detection when compared with subjects with average-risk (OR, 2.00; 95\% CI 1.17-3.41) (Table 4).

Combination between overweight and APCS scoring system

With the combination of overweight and the APCS score, subjects were classified into 4 groups; 36 subjects $(11 \%)$ were categorized as high-risk group with

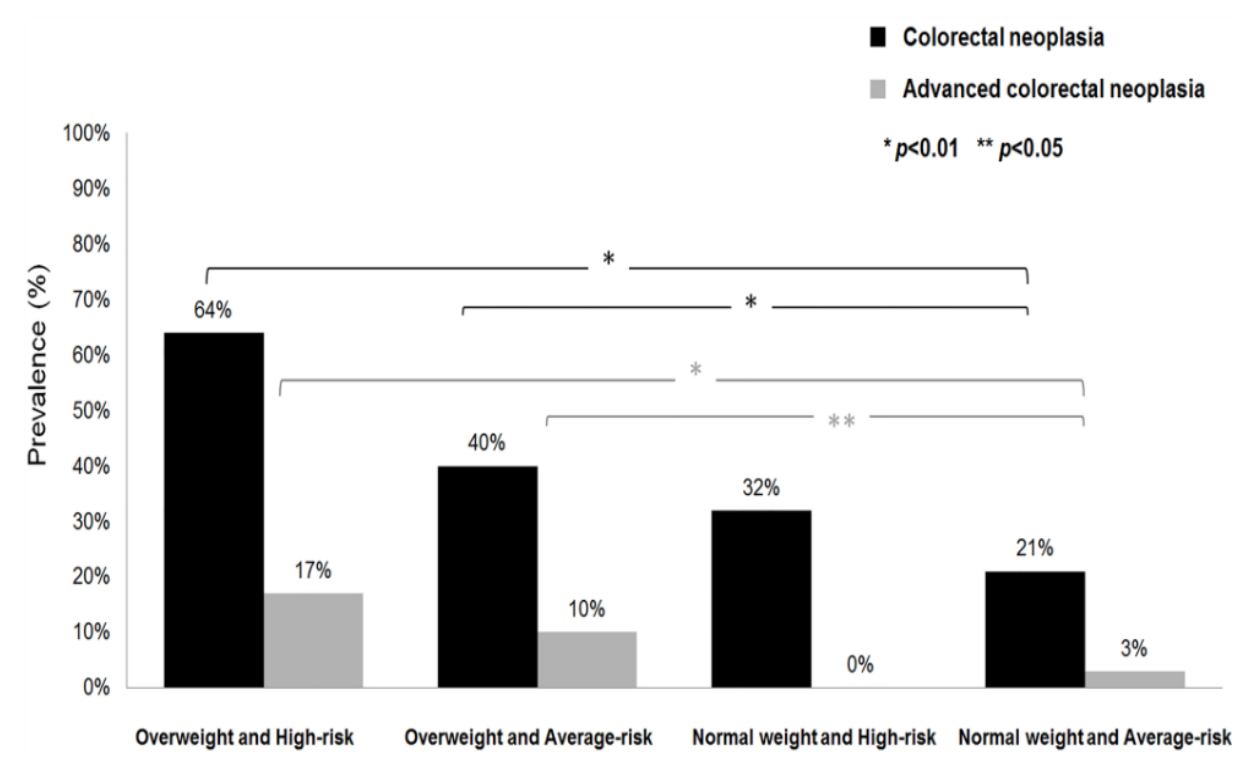

Figure 1. Prevalence of Colorectal Neoplasia and Advanced Colorectal Neoplasia Classified by Overweight and Asia-Pacific Colorectal Scoring System 
Table 3. Univariate and Multivariate Analysis for the Association between Potential Risk Factors and the Detection Rate of Colorectal Neoplasia

\begin{tabular}{|c|c|c|c|c|c|}
\hline \multirow[t]{2}{*}{ Model 1 ${ }^{\mathrm{a}}$} & \multirow{2}{*}{$\begin{array}{c}\text { Colorectal neoplasia }(\mathrm{n}=120) \\
\quad(\text { prevalence, } \%)\end{array}$} & \multicolumn{2}{|c|}{ Univariate analysis } & \multicolumn{2}{|c|}{ Multivariate analysisc } \\
\hline & & Odds ratio $(95 \% \mathrm{CI})$ & $\mathrm{p}$-value & Odds ratio $(95 \% \mathrm{CI})$ & p-value \\
\hline Age (per 1-year increase) & & $1.03(1.00-1.05)$ & 0.05 & $1.03(1.00-1.06)$ & 0.05 \\
\hline \multicolumn{6}{|l|}{ Sex } \\
\hline Male $(n=129)$ & $57(44 \%)$ & $1.84(1.16-2.90)$ & $<0.01$ & $1.61(1.00-2.59)$ & 0.05 \\
\hline Female $(\mathrm{n}=209)$ & $63(30 \%)$ & reference & & reference & \\
\hline \multicolumn{6}{|c|}{ First-degree relative with CRC } \\
\hline Present $(n=28)$ & $16(57 \%)$ & $2.64(1.21-5.79)$ & 0.02 & $2.95(1.29-6.72)$ & 0.01 \\
\hline Absent $(n=310)$ & $104(36 \%)$ & reference & & reference & \\
\hline Smoking & & & & -- & -- \\
\hline Current/former $(n=39)$ & $15(39 \%)$ & $1.16(0.58-2.30)$ & 0.68 & & \\
\hline Never $(n=299)$ & $105(35 \%)$ & reference & & & \\
\hline \multicolumn{6}{|l|}{ Body mass index } \\
\hline$\geq 23 \mathrm{~kg} / \mathrm{m}^{2}(\mathrm{n}=192)$ & $85(44 \%)$ & $2.52(1.57-4.05)$ & $<0.01$ & $2.57(1.57-4.20)$ & $<0.01$ \\
\hline$<23 \mathrm{~kg} / \mathrm{m}^{2}(\mathrm{n}=146)$ & $35(24 \%)$ & reference & & reference & \\
\hline \multicolumn{6}{|l|}{ Metabolic syndrome } \\
\hline Present $(n=136)$ & $52(38 \%)$ & $1.22(0.78-1.92)$ & 0.39 & -- & -- \\
\hline Absent $(\mathrm{n}=202)$ & $68(34 \%)$ & reference & & & \\
\hline \multicolumn{6}{|l|}{ Model $2^{b}$} \\
\hline \multicolumn{6}{|l|}{ APCS score } \\
\hline High risk $(\mathrm{n}=70)$ & $34(49 \%)$ & $2.00(1.17-3.41)$ & 0.01 & $2.23(1.28-3.88)$ & $<0.01$ \\
\hline Average risk $(\mathrm{n}=268)$ & $86(32 \%)$ & reference & & reference & \\
\hline \multicolumn{6}{|l|}{ Body mass index } \\
\hline$\geq 23 \mathrm{~kg} / \mathrm{m}^{2}(\mathrm{n}=192)$ & $85(44 \%)$ & $2.52(1.57-4.05)$ & $<0.01$ & $2.68(1.65-4.36)$ & $<0.01$ \\
\hline$<23 \mathrm{~kg} / \mathrm{m}^{2}(\mathrm{n}=146)$ & $35(24 \%)$ & reference & & reference & \\
\hline \multicolumn{6}{|l|}{ Metabolic syndrome } \\
\hline Present $(n=136)$ & $52(38 \%)$ & $1.22(0.78-1.92)$ & 0.39 & -- & -- \\
\hline Absent $(n=202)$ & $68(34 \%)$ & reference & & & \\
\hline
\end{tabular}

Table 4. Prevalence of Colorectal Neoplasia According to Overweight, APCS Score and the Combination of Overweight and the APCS Score

\begin{tabular}{|c|c|c|c|}
\hline \multirow[t]{2}{*}{ All subjects $(\mathrm{n}=338)$} & \multicolumn{3}{|c|}{ Colorectal neoplasia } \\
\hline & Prevalence & Odds ratio $(95 \% \mathrm{CI})$ & p-value \\
\hline \multicolumn{4}{|l|}{ Overweight } \\
\hline Present $(n=192)$ & $85(44 \%)$ & $2.52(1.57-4.05)$ & $<0.01$ \\
\hline Absent $(\mathrm{n}=146)$ & $35(24 \%)$ & reference & \\
\hline \multicolumn{4}{|l|}{ APCS scoring system } \\
\hline High risk $(\mathrm{n}=70)$ & $34(49 \%)$ & $2.00(1.17-3.41)$ & 0.01 \\
\hline Average risk $(\mathrm{n}=268)$ & $86(32 \%)$ & reference & \\
\hline \multicolumn{4}{|l|}{ APCS scoring system and overweight } \\
\hline G1: overweight and high risk $(\mathrm{n}=36)$ & $23(64 \%)$ & $6.49(2.87-14.67)$ & $<0.01$ \\
\hline G2: overweight and average risk $(n=156)$ & $62(40 \%)$ & $2.42(1.39-4.21)$ & $<0.01$ \\
\hline G3: normal weight and high risk $(n=34)$ & $11(32 \%)$ & $1.75(0.75-4.10)$ & 0.19 \\
\hline G4: normal weight and average risk $(n=112)$ & $24(21 \%)$ & reference & -- \\
\hline
\end{tabular}


overweight (G1), 156 subjects (46\%) were categorized as average-risk group with overweight (G2), 34 subjects $(10 \%)$ were categorized as high-risk group with normal weight (G3), and 112 subjects (33\%) were categorized as average-risk group with normal weight (G4). The detection rates of CRN for G1, G2, G3 and G4 were $64 \%$, $40 \%, 32 \%$ and $21 \%$, respectively $(\mathrm{p}<0.01)$. One CRC was found in G1 and one CRC was found in G2. None of CRC was detected in G3 and G4. The significant differences in the detection rates of advanced CRN among 4 groups was observed $(\mathrm{p}<0.01)$ (Figure 1). For the risk of CRN detection, the OR for G1 was 6.49 (95\% CI, 2.87-14.67), 2.42 for G2 (1.39-4.21) and 1.75 for G3 (0.75-4.10) as compared with G4 (Table 4).

\section{Discussion}

In this study, we demonstrated that apart from the traditional CRC risk factors, overweight is the additional risk factor for CRN detection in asymptomatic subjects. In other words, the synergistic effect of combining overweight with the APCS score for predicting the CRN detection was observed. In subjects with high-risk, the relative risk for detecting $\mathrm{CRN}$ were twice as likely as subjects with average-risk. Likewise, in subjects with overweight, the risk for detecting CRN increased to 2.5-fold as compared to subjects with normal weight. Synergistically, high-risk subjects with overweight had approximately 6.5-fold increased risk for detecting CRN as compared to average-risk subjects with normal weight.

APCS scoring system is helpful to predict individual harboring $\mathrm{CRN}$ in Asian population. In this study, the result is concordant with our earlier reports showing an approximately 1.5 -fold higher prevalence of colorectal adenoma in the high-risk subjects as compared to that of in the average-risk subjects (Aniwan et al., 2015; Aniwan et al., 2017). The development of APCS scoring system included several important variables including age, sex, smoking, family history of CRC, alcohol and diabetes. However, there were some limitations in the APCS score, other potential clinical factors regarding component of metabolic syndrome (i.e. body mass index, hypertension, and hyperlipidemia) were not included during the initial development of the APCS score (Yeoh et al., 2011). In this study, BMI and metabolic syndrome were combined with individual parameter of APCS score and later assessed. Moreover, the separate analysis on the risk stratified by APCS was also performed. A recent study from Hong Kong developed a modified APCS score in Chinese population by adding BMI $\geq 23 \mathrm{~kg} / \mathrm{m}^{2}$ as 1 point and deducting 1 point form age and family history criteria (Sung et al., 2018). The new model showed that the modified high-risk subjects had 2-fold increased risk for CRN and 2.5-fold increased risk for advanced CRN as compared with the modified low-risk subjects (Sung et al., 2018). However this modified APCS score requires further validation by comparing with the original APCS score.

The objective of using risk stratified approach for CRC screening is to minimize the burden of colonoscopy and maximize the detection rate of CRN. Accordingly, the approach strategy is based on the prevalence of CRN and the number of requiring colonoscopy to detect CRN. Theoretically, selecting subjects with higher risk for CRN to undergo for colonoscopy helps to reduce the number of colonoscopy needed to detect one CRN, whereas the lower risk subjects should undergo for a colonoscopy only after being screened by a less expensive test with higher predictive value. Herein, our results showed that the prevalence of CRN were $36 \%$ in all screened subjects, $49 \%$ in the high-risk subjects, and $64 \%$ in the high-risk subjects with overweight, respectively. The number needed for colonoscopy (NNC) to detect one CRN in all screened subjects was 2.8 whereas the NNC to detect one $\mathrm{CRN}$ in subjects with high APSC score was 2, which corresponded to $29 \%$ reduction rate of colonoscopy. Incorporating BMI with the APCS score, the NNC to detect one $\mathrm{CRN}$ in high-risk subjects with overweight was even lower (1.6), and resulted to $43 \%$ reduction in NNC.

With regard to the association between BMI and the risk of CRN, our results are in line with previous studies and meta-analyses (Okabayashi et al., 2012; Omata et al., 2013). Okabayashi et al., (2012) demonstrated a significant association between an increased BMI and CRN. The prevalence of colorectal adenoma in subjects with overweight defined as BMI of $25-30 \mathrm{~kg} / \mathrm{m}^{2}$ was $21 \%$ higher than those of in subjects with BMI $<25 \mathrm{~kg} / \mathrm{m}^{2}$ while the prevalence of colorectal adenoma in obesity (BMI $\geq 30 \mathrm{~kg} / \mathrm{m}^{2}$ ) was $32 \%$ higher when compared to those with BMI $<25 \mathrm{~kg} / \mathrm{m}^{2}$. It is noted that in our study, we defined overweight as BMI $\geq 23 \mathrm{~kg} / \mathrm{m}^{2}$. Because Thais have low rates of overweight/obesity defined by the standard definition for Asian population according to WHO. In 2003, by using this definition, one-third of Thai population had overweight and less than $10 \%$ with obesity whereas two-third of United States population had overweight and more than $20 \%$ with obesity (WHO, 2004). However, the prevalence of CRN at the cutoff level of BMI $23 \mathrm{~kg} / \mathrm{m}^{2}$ in our study still held true by showing the higher prevalence than those with lower BMI. An additional support from Chinese population confirmed that the prevalence of CRN and advanced CRN were comparable between using the cutoff level of BMI $\geq 23 \mathrm{~kg} / \mathrm{m}^{2}(35 \%$ and $6.5 \%)$ and the cutoff level of BMI $\geq 25 \mathrm{~kg} / \mathrm{m}^{2}$ (38\% and $7 \%$ ), respectively (Sung et al., 2018). In addition, This study showed that using the cutoff level of BMI $\geq 23 \mathrm{~kg} / \mathrm{m}^{2}$ had enough power to detect more advanced CRN (OR, 1.5; 95\% CI, 1.1-2.1) similar to using the higher cutoff $\mathrm{BMI} \geq 25 \mathrm{~kg} /$ $\mathrm{m}^{2}$ (OR, 1.4; 1.1-1.9) (Sung et al., 2018). Another study from Korea supports that there was no difference between using the cutoff level of BMI $23 \mathrm{~kg} / \mathrm{m}^{2}$ and BMI $25 \mathrm{~kg} /$ $\mathrm{m}^{2}$ on the risk of CRC (Shin et al., 2017). The authors showed that when compared to those with BMI at 18.5-23 $\mathrm{kg} / \mathrm{m}^{2}$, the relative risk of CRC increased to $17 \%$ equally in those with BMI $23-25 \mathrm{~kg} / \mathrm{m}^{2}$ and those with BMI $25-30$ $\mathrm{kg} / \mathrm{m}^{2}$ (Shin et al., 2017).

A previous meta-analysis study from worldwide populations demonstrated there was a modest association between metabolic syndrome and the risk of CRC; $33 \%$ increased risk of $\mathrm{CRC}$ for men and $41 \%$ increased risk of CRC for women (Esposito et al., 2013). However, subgroup analysis by race showed no increased risk of $\mathrm{CRC}$ in Asian populations with metabolic syndrome

Asian Pacific Journal of Cancer Prevention, Vol 20 
(Esposito et al., 2013). Their results were similar to our findings. We found that subjects with metabolic syndrome had $22 \%$ increase in the risk for detecting $\mathrm{CRN}$ as compared to those without metabolic syndrome, unfortunately this was not significant statistically. Perhaps metabolic syndrome has a lower impact to predict the risk for CRN as compared to the BMI factor, especially in Asian populations. However, we did not perform a separate analysis by metabolic syndrome component such as abdominal girth, triglyceride and fasting plasma sugar levels. Because there was a relatively small number of CRN among metabolic syndrome component in this study. Therefore no conclusion regarding to these components could be drawn.

A key strength of this study is that all asymptomatic subjects were prospectively enrolled. Data on body weight, height, component of metabolic syndrome were measured rather than using patient-reported. However, there are certain limitations in this study. First, all subjects were enrolled from the CRC screening clinic. Therefore, we cannot avoid self-referral bias. This could be the explanation that we had more than half of our screened subjects were female (62\%) and being an overweight $(57 \%)$. Possibly, women often are interested in healthrelated information and subjects being overweight are the population that already caught medical attention for their metabolic syndrome. Second, our CRC screening subjects were recruited either from those who presented for opportunistic colonoscopy or from those with positive fecal immunochemical test (FIT). This could explain the high prevalence of CRC was observed in our population. The selection bias would limit the generalizability of our results. By prediction, colonoscopy in subjects with FIT positive would have a higher prevalence of CRN than that of from those without prior FIT screening (Aniwan et al., 2015). However, the overall prevalence of CRN in our study was $36 \%$ and this was not higher than the previous report in opportunistic colonoscopies (Aniwan et al., 2015). Third, we used different cutoff level of BMI. This might possibly restrict the generalizability to the countries with higher percentage of obese population. However, in the Asia-Pacific region, the lower cutoff level of BMI could be more practical and appropriate to combine with the Asian risk score. Lastly, our results showed higher prevalence of CRN in overweighed highrisk population as compared with normal weight high-risk population but was not statistically significant which may probably caused by the relatively small number of CRN in our subgroups. In conclusion, this study illustrates that in addition to APCS scoring system, overweight is an independent risk factor for detecting CRN. A combination of overweight and the APCS score is useful for improving the prediction for $\mathrm{CRN}$ and may prioritize patients for colonoscopy in a country with limited resource. However, the slightly lower BMI cutoff for determining overweight used in our study may be applicable only to the countries with low prevalence of obese population.

\section{Abbreviations \\ APCS: Asia-Pacific Colorectal Screening \\ BMI: body mass index}

CI: confidence interval

CRC: colorectal cancer

CRN: colorectal neoplasia

FIT: fecal immunochemical test

HDL: high-density lipoproteins

OR: odds ratio

\section{Grant support}

This research was supported by Chulalongkorn University, Grant for International Research Integration: Chula Research Scholar, Ratchadaphiseksomphot Endowment Fund.

\section{Conflicts of interests}

None of the authors have any relevant conflicts of interests.

\section{References}

Aniwan S, Orkoonsawat P, Viriyautsahakul V, et al (2016). The secondary quality indicator to improve prediction of adenoma miss rate apart from adenoma detection rate. Am J Gastroenterol, 111, 723-9.

Aniwan S, Ratanachu-Ek T, Pongprasobchai S, et al (2017). Impact of fecal $\mathrm{Hb}$ levels on advanced neoplasia detection and the diagnostic miss rate for colorectal cancer screening in high-risk vs. Average-risk subjects: a multi-center study. Clin Transl Gastroenterol, 8, e113.

Aniwan S, Rerknimitr R, Kongkam P, et al (2015). A combination of clinical risk stratification and fecal immunochemical test results to prioritize colonoscopy screening in asymptomatic participants. Gastrointest Endosc, 81, 719-27.

Braun S, Bitton-Worms K, LeRoith D (2011). The link between the metabolic syndrome and cancer. Int J Biol Sci, 7, 1003-15.

Byrne AM, Bouchier-Hayes DJ, Harmey JH (2005). Angiogenic and cell survival functions of vascular endothelial growth factor (VEGF). J Cell Mol Med, 9, 777-94.

Corte C, Zhang L, Chen J, et al (2016). Validation of the Asia Pacific Colorectal Screening (APCS) score in a Western population: An alternative screening tool. J Gastroenterol Hepatol, 31, 370-5.

Esposito K, Chiodini P, Capuano A, et al (2013). Colorectal cancer association with metabolic syndrome and its components: a systematic review with meta-analysis. Endocrine, 44, 634-47.

Harriss DJ, Atkinson G, George K, et al (2009). Lifestyle factors and colorectal cancer risk (1): systematic review and meta-analysis of associations with body mass index. Colorectal Dis, 11, 547-63.

Hursting SD, Hursting MJ (2012). Growth signals, inflammation, and vascular perturbations: mechanistic links between obesity, metabolic syndrome, and cancer. Arterioscler Thromb Vasc Biol, 32, 1766-70.

Kelly T, Yang W, Chen CS, et al (2008). Global burden of obesity in 2005 and projections to 2030. Int J Obes (Lond), 32, 1431-7.

National Cholesterol Education Program Expert Panel on Detection EToHBCi, Adults (2002). Third report of the National Cholesterol Education Program (NCEP) expert panel on detection, evaluation, and treatment of high blood cholesterol in adults (Adult treatment panel III) final report. Circulation, 106, 3143-421.

Okabayashi K, Ashrafian H, Hasegawa H, et al (2012). Body mass index category as a risk factor for colorectal adenomas: 
a systematic review and meta-analysis. Am J Gastroenterol,

107, 1175-85; quiz 86.

Omata F, Deshpande GA, Ohde S, et al (2013). The association between obesity and colorectal adenoma: systematic review and meta-analysis. Scand J Gastroenterol, 48, 136-46.

Pollak M (2008). Insulin and insulin-like growth factor signalling in neoplasia. Nat Rev Cancer, 8, 915-28.

Quach DT, Hiyama T, Nguyen TA, et al (2018). Asia-Pacific Colorectal Screening score: A useful tool to stratify risk for colorectal advanced neoplasms in Vietnamese patients with irritable bowel syndrome. J Gastroenterol Hepatol, 33, 150-5.

Shin CM, Han K, Lee DH, et al (2017). Association among obesity, metabolic health, and the risk for colorectal cancer in the general population in Korea using the National Health Insurance Service-National sample cohort. Dis Colon Rectum, 60, 1192-200.

Stattin P, Lukanova A, Biessy C, et al (2004). Obesity and colon cancer: does leptin provide a link?. Int J Cancer, 109, 149-52.

Sung JJ, Lau JY, Goh KL, et al (2005). Increasing incidence of colorectal cancer in Asia: implications for screening. Lancet Oncol, 6, 871-6.

Sung JJ, Ng SC, Chan FK, et al (2015). An updated Asia Pacific Consensus Recommendations on colorectal cancer screening. Gut, 64, 121-32.

Sung JJY, Wong MCS, Lam TYT, et al (2018). A modified colorectal screening score for prediction of advanced neoplasia: A prospective study of 5744 subjects. $J$ Gastroenterol Hepatol, 33, 187-94.

WHO Eexpert Consultation (2004). Appropriate body-mass index for Asian populations and its implications for policy and intervention strategies. Lancet, 363, 157-63.

Yeoh KG, Ho KY, Chiu HM, et al (2011). The Asia-Pacific Colorectal Screening score: a validated tool that stratifies risk for colorectal advanced neoplasia in asymptomatic Asian subjects. Gut, 60, 1236-41.

\section{c) (7) (8)}

This work is licensed under a Creative Commons AttributionNon Commercial 4.0 International License. 